\title{
Competition between multiple totally asymmetric simple exclusion processes for a finite pool of resources
}

\author{
L. Jonathan Cook, ${ }^{*}$ R. K. P. Zia, ${ }^{\dagger}$ and B. Schmittmann \\ Department of Physics, Virginia Tech, Blacksburg, Virginia 24061, USA
}

(Received 22 June 2009; published 28 September 2009)

\begin{abstract}
Using Monte Carlo simulations and a domain-wall theory, we discuss the effect of coupling several totally asymmetric simple exclusion processes (TASEPs) to a finite reservoir of particles. This simple model mimics directed biological transport processes in the presence of finite resources such as protein synthesis limited by a finite pool of ribosomes. If all TASEPs have equal length, we find behavior which is analogous to a single TASEP coupled to a finite pool. For the more generic case of chains with different lengths, several unanticipated regimes emerge. A generalized domain-wall theory captures our findings in good agreement with simulation results.
\end{abstract}

DOI: 10.1103/PhysRevE.80.031142

PACS number(s): 02.50.- r, 05.60.-k

\section{INTRODUCTION}

A fundamental and comprehensive understanding of nonequilibrium phenomena remains one of the greatest challenges of current condensed-matter and materials physics [1], with significant consequences for advances in materials science, the life sciences, and engineering. Even the nonequilibrium steady states of open, transport-carrying systems continue to defy our equilibrium-trained expectations and intuitions. One approach toward progress has focused on investigating simple model systems with the goal of identifying generic classes of behaviors.

The totally asymmetric simple exclusion process (TASEP) $[2,3]$ is one of these models. It has acquired paradigmatic status for several reasons: (i) it is very simple, consisting of particles hopping along a one-dimensional chain; (ii) with open boundaries, it shows highly nontrivial behaviors such as distinct phases, shocks, and long-range correlations in both space and time; (iii) in its simplest forms, its steady-state properties, as well as selected dynamic quantities, can be found exactly; and finally, (iv) the model is closely related to interesting applications such as biological transport [4,5] or traffic flow [6]. At the origin of this richness lies the violation of detailed balance. The specific behaviors depend strongly on the boundary conditions. With periodic boundary conditions, the stationary state is a flat distribution with all configurations equally probable [7]. However, the dynamics of this system is nontrivial and differs from simple diffusion [8]. With open boundary conditions, particles are injected at one end and removed at the other with different (but constant) rates. In this case, even the steady state is nontrivial and remained unknown for two decades [9-11]. Despite being a one-dimensional system with short-range interactions and dynamics, the open TASEP displays distinct (stationary) phases [12] controlled by the entrance and exit rates. As may be expected, the dynamic properties are even more complex and rich [13-16].

\footnotetext{
*lacook1@vt.edu

†rkpzia@vt.edu

†schmittm@vt.edu
}

These TASEP studies have recently been extended by coupling the chain to a finite (rather than infinite) particle reservoir $[17,18]$ reflecting a constraint on the total number of particles. In [17], the particles represent cars leaving a parking garage, so that the rate of entry onto the roadway (the lattice) is chosen to be a constant, as long as there is at least one car in the garage. In [18], the TASEP models a biological transport process [4] and the constraint reflects the finite number of ribosomes in a cell, with those leaving the mRNA (the lattice) being "recycled" to the entry point. The origins and effects of "ribosome recycling" are multifaceted, such as diffusion of the recycled components from termination to initiation sites [19]. Addressing all possible issues for a real cell is beyond the scope of this study and our aim here is modest, namely, to explore how the finite pool of available particles affects the lattice occupation and currents. As in [18], we consider an entry rate that depends smoothly on the number of particles in the pool, $N_{p}$. In particular, we choose a rate function which is proportional to $N_{p}$ when the concentrations of ribosomes in the cell are limited, and, when particles are abundant, becomes a constant-the inherent binding rate of a ribosome. The simulation results for a single TASEP can be described well by a domain-wall (DW) theory $[14,15,18]$ especially when generalized to account for the feedback [20].

In addition to being constrained to a finite pool of ribosomes in a living cell, a mRNA must compete against many other different genes (or mRNAs) for these resources. Therefore, we are motivated to study the competition of multiple TASEPs for the same pool of particles. Since different mRNAs are comprised of different numbers of codons, it is reasonable to study TASEPs with different lengths. Does one "win" and another one "lose"? What does "winning" and "losing" mean?

This paper is organized as follows. The next section introduces our model. In Sec. III, we present simulation results for two and three TASEPs connected to a finite reservoir of particles. Analytic results, based on our generalized DW theory [20], are derived for an arbitrary collection of TASEPs and will be discussed in Sec. IV. A final section is devoted to a summary and outlook. 


\section{MODEL FOR COMPETING TASEPS}

Let us first review the standard (or "ordinary") TASEP. Each site of a discrete lattice of length $L$ is labeled by $i$ $=1, \ldots, L$ and may be empty or occupied by a single particle. Thus, a particular configuration can be described by a set of occupation numbers, $\{n(i)\}$, each taking the values 0,1 . A configuration evolves to a new one according to the following rules. A particle is chosen at random, say, at site $i$. If the neighboring site to its right (site $i+1)$ is empty, the particle hops there with rate unity. If the particle is located on the last site $(i=L)$ of the lattice, it exits with a probability $\beta$. In addition to the particles on the lattice, we assign a "virtual particle" to label an external reservoir, so that when chosen, a particle is placed with probability $\alpha$, on the first site $(i=1)$ of the lattice, provided $n(1)=0$. Notice that this system can be regarded as a total of $N_{\text {tot }}$ particles hopping on a ring with $L+1$ sites, where the site $i=0$ is associated with the reservoir and can be occupied by any number of particles. Of course, the hopping rates into and out of this site are different from the rest. They can be represented, respectively, as $\beta n(L)$ and $\alpha[1-n(1)]$. Note that neither depends on $n(0)$ and so, as long as $N_{t o t}>L$, there would be at least one particle which can be injected onto the lattice. Indeed, this is the scenario presented in the parking garage problem [17], in which interesting transitions are studied for $N_{t o t} \in[0, L]$. Lastly, the seemingly complicated rule of choosing real and virtual particles can be replaced, in this formulation of TASEP, by "randomly choosing an occupied site."

In the steady state, this simple model exhibits three phases: a low-density (LD) phase for $\alpha<1 / 2$ and $\alpha<\beta$, a high-density (HD) phase for $\beta<1 / 2$ and $\beta<\alpha$, and a maximal current (MC) phase for $\alpha, \beta>1 / 2$. The densities $\bar{\rho}$ in the three phases are given by $\alpha(\mathrm{LD}), 1-\beta$ (HD), and $1 / 2(\mathrm{MC})$, respectively. The line $\alpha=\beta<1 / 2$ marks the coexistence of $\mathrm{HD}$ and LD phases and is characterized by the presence of a shock which performs an unbiased random walk through the whole lattice. For this reason, the coexistence line is also sometimes referred to as the "shock phase" (SP). Since the entrance and exit rates are constant here, $N_{\text {tot }}$ plays no role and can be finite (but larger than $L$ ) instead of $\infty$.

Our motivation for studying the TASEP is modeling protein synthesis in a cell [4], in which the particles represent ribosomes. Thus, $N_{t o t}$ is finite and must be shared between many mRNA's (there may be as many as 10000 mRNAs in some cells). Only the unbound ribosomes (i.e., those in the "pool," totaling $N_{p}$ ) are available for initiation (i.e., to enter any one TASEP). Assuming the concentration of these ribosomes is uniform, we introduced a more realistic model [18] for the entry rate, $\alpha$, that depends on $N_{p}$ as follows. Starting with just one lattice (mRNA) in our system, let us denote its total occupation by $N$, so that

$$
N_{\text {tot }}=N+N_{p}
$$

is kept fixed. Particles still exit the lattice as before, with rate $\beta$, and are placed immediately into the pool. In contrast to the ordinary TASEP, the (effective) entry rate, $\alpha_{\text {eff }}$, will now be controlled by $N_{p}$ through $\alpha_{e f f}=\alpha f\left(N_{p}\right)$. Here, $\alpha$ is a constant and $f\left(N_{p}\right)$ assumes a value in $[0,1]$ for all $N_{p}$. Physi- cally, $\alpha_{\text {eff }}$ should vanish if there are no particles in the pool, whence we impose $f(0)=0$. Further, $f$ should be a monotonically increasing smooth function of $N_{p}$. Finally, for sufficiently large $N_{p}$, we should recover the standard TASEP with a constant entry rate, $\alpha$, whence $\lim _{N_{p} \rightarrow \infty} f\left(N_{p}\right)=1$. These properties are motivated by the notion that the rate for a ribosome to bind to the mRNA (a) should be limited by the ribosome availability, especially for low concentrations and (b) should approach some intrinsic rate for the binding process, when the ribosomes are abundant. The specific form of $f\left(N_{p}\right)$ is not very important but we follow [18] and use

$$
f\left(N_{p}\right)=\tanh \left(N_{p} / N^{*}\right),
$$

where $N^{*}$ is a suitably defined normalization, or crossover, scale. In [18], $N^{*}$ was chosen to be the average number of particles for the standard TASEP with entry and exit rates $\alpha$ and $\beta$.

In the following, we will consider multiple open TASEPs of various lengths, $L_{\ell}(\ell=1,2, \ldots, M), M$ being the total number of chains in our system. Writing the occupation in each as $N_{\ell}$, we naturally define

$$
\rho_{\ell} \equiv N_{\ell} / L_{\ell}
$$

as the overall densities on each chain and of course,

$$
N_{\text {tot }}=\sum_{\ell=1}^{M} N_{\ell}+N_{p}
$$

as the generalization of Eq. (1). Most of our simulation results are for $M=2$, with a few for $M=3$. All $M$ TASEPs draw their particles from the same reservoir, according to the same $\alpha_{e f f}=\alpha f\left(N_{p}\right)$ with $f$ given by Eq. (2). For multiple chains of different lengths, we choose $N^{*}$ to be

$$
N^{*}=M^{-1} \sum_{\ell=1}^{M} \bar{\rho}_{\ell} L_{\ell},
$$

where $\bar{\rho}_{\ell}$ is the average density for an ordinary TASEP of length $L_{\ell}$, with entry and exit rates $\alpha$ and $\beta$. This choice of $N^{*}$ only serves to locate the specific value of $N_{\text {tot }}$ at which the system crosses a phase boundary. It also allows us to define (somewhat arbitrarily)

$$
\rho_{t o t} \equiv N_{t o t} / M^{-1} \sum_{\ell=1}^{M} L_{\ell} .
$$

For our simulation studies, we define a Monte Carlo step (MCS) as making $M+\sum L_{\ell}$ attempts to choose a site to update. Note that, on the average, the pool is updated $M$ times as often as a site on any lattice. This choice corresponds to updating all links (nearest-neighbor pairs of sites) with equal probability, with the understanding that each TASEP is linked to the pool (independently of the others). In this study, the entry rates to all chains are chosen to be the same $\alpha_{\text {eff }}$. We typically start with all particles in the pool and wait $100 \mathrm{k}$ MCS for the system to reach steady state. Every 100 MCS thereafter, we measure the site occupations, $n(i)$, in each chain. Using up to 10k measurements as our "ensemble," we compute the density profile 


$$
\rho(i) \equiv\langle n(i)\rangle
$$

and the overall density $\rho \equiv \Sigma_{i} \rho(i) / L$ for each chain. The average steady-state current, denoted by $J$, is obtained by monitoring the total number of particles that enter (and exit) a chain over the last $1 \mathrm{M}$ MCS. Note that $J$ is also just the product $\beta \rho(L)$.

\section{SIMULATION RESULTS}

In this section, we present simulation data for the model described above. In principle, any number of TASEPs can be connected to the pool. However, to gain some insight into competition, we begin with the simplest case, with only two TASEPs. Although some selected results for the $M=3$ case will be presented at the end, most of our data here is for the case of $M=2$. As we will see, features already appear when just one more TASEP is added to the system. By contrast, we have not encountered, so far, any further surprising phenomena by considering $M>2$. As in the earlier study [18], we explore four regimes here, with $\alpha$ and $\beta$ corresponding to the $\mathrm{LD}, \mathrm{HD}$, and MC phases of the ordinary TASEP, as well as the coexistence line, SP. Our main interest is how an increasing $N_{\text {tot }}$ affects the various profiles and so, the average overall densities and currents.

\section{A. LD phase}

When $\alpha<1 / 2$ and $\alpha<\beta$, the standard TASEP displays the LD phase. If coupled to a finite pool, it remains in the LD phase, since $\alpha_{e f f} \leq \alpha$. The only difference is that the density and current of the system rise linearly with $N_{\text {tot }}$ for small values of $N_{\text {tot }}$ and approach their asymptotic values from below as $N_{t o t} \rightarrow \infty$. When two TASEPs compete for this finite pool of resources, similar behavior is found. The results for equal length chains are illustrated in Fig. 1(a) for $\alpha=0.3, \beta$ $=0.7$, and $L_{1}=L_{2}=1000$. As $N_{\text {tot }}$ is increased, the two TASEPs both remain in the LD phase, with equal densities, $\rho_{1}=\rho_{2}=\rho$, and currents, $J_{1}=J_{2}=J$. Since both TASEPs are controlled by the same $\alpha_{\text {eff }}$ and $\beta$, there is full symmetry between the two and the observed behavior is hardly remarkable. The only notable difference between our system and the one with a single constrained TASEP is observed in $\rho\left(N_{t o t}\right)$ for $N_{t o t} \ll \alpha L$. For our case, $\rho \rightarrow \rho_{t o t} / 3$ as opposed to $\rho_{t o t} / 2$ for the single TASEP. This property is easily understandable, since we can regard the pool as an additional "chain" and note that the resources are evenly distributed among three (or two) chains. For large $N_{\text {tot }}$, both TASEP densities level off to $\alpha$, of course. Slightly more interesting is the case where the two chains have very different lengths such as $L_{1}=1000$ and $L_{2}=100$. Due to the lack of symmetry, it is less obvious that $\rho_{1}=\rho_{2} \rightarrow \rho_{\text {tot }} / 3$ is still valid for small $\rho_{\text {tot }} \ll \alpha$. However, it is straightforward to show, using the methods in previous studies $[18,20]$, that $\rho_{1}=\ldots=\rho_{M} \rightarrow \rho_{t o t} /(M+1)$ in general, given the specific form of $\alpha_{e f f}\left(N_{p}\right)$ we chose. At the opposite limit, the approach to the asymptotic regime is somewhat faster than for the equal length case. This behavior is also expected, since the "total" system size $\left(L_{1}+L_{2}=1100\right)$ is considerably smaller than the case above $\left(L_{1}+L_{2}=2000\right)$, so that saturation sets in at smaller values of $N_{\text {tot }}$.
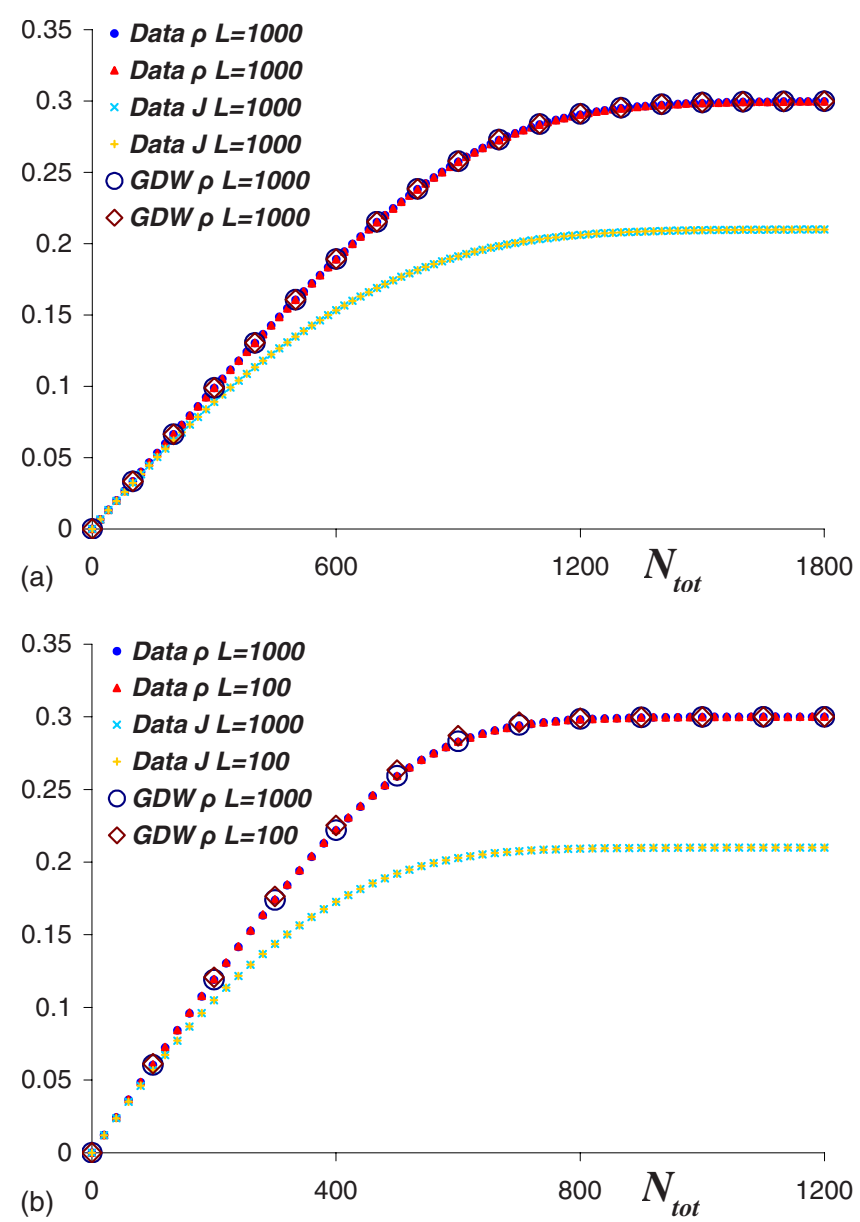

FIG. 1. (Color online) Average overall density and current as a function of $N_{\text {tot }}$ for (a) $L_{1}=L_{2}=1000$ and (b) $L_{1}=1000$ and $L_{2}=100$ with $\alpha=0.3$ and $\beta=0.7$.

To summarize, the overall densities of competing chains behave just as a single TASEP coupled to a finite pool of particles. Meanwhile, the overall currents are, except for finite-size effects, also essentially the same: $\rho(1-\rho)$.

\section{B. MC phase}

For a single TASEP coupled to a finite pool with $\alpha, \beta$ $>1 / 2$, the current approaches its asymptotic value (1/4) smoothly from below. However, the density exhibits a sharp "kink," which marks the crossing of the LD-MC phase boundary when $N_{\text {tot }}$ becomes large enough to sustain a density of $1 / 2$ on the chain. The range of $N_{\text {tot }}$ over which this crossover occurs is controlled by the system size: For large system sizes, it becomes very narrow and, therefore, appears as a kink; in smaller systems, the crossover occurs over a larger range and appears smooth. For the case of two TASEPs, we observe very similar behavior. Figure 2 shows our data for $\alpha=\beta=0.7$ and $L_{1}=L_{2}=1000$. Again, the currents and densities of the two TASEPs coincide provided the two TASEPs have equal lengths. For unequal lengths, we observe the anticipated finite-size effect in the density: the longer TASEP displays a much sharper crossover from LD to MC behavior than the shorter one. Like the LD case, the overall 

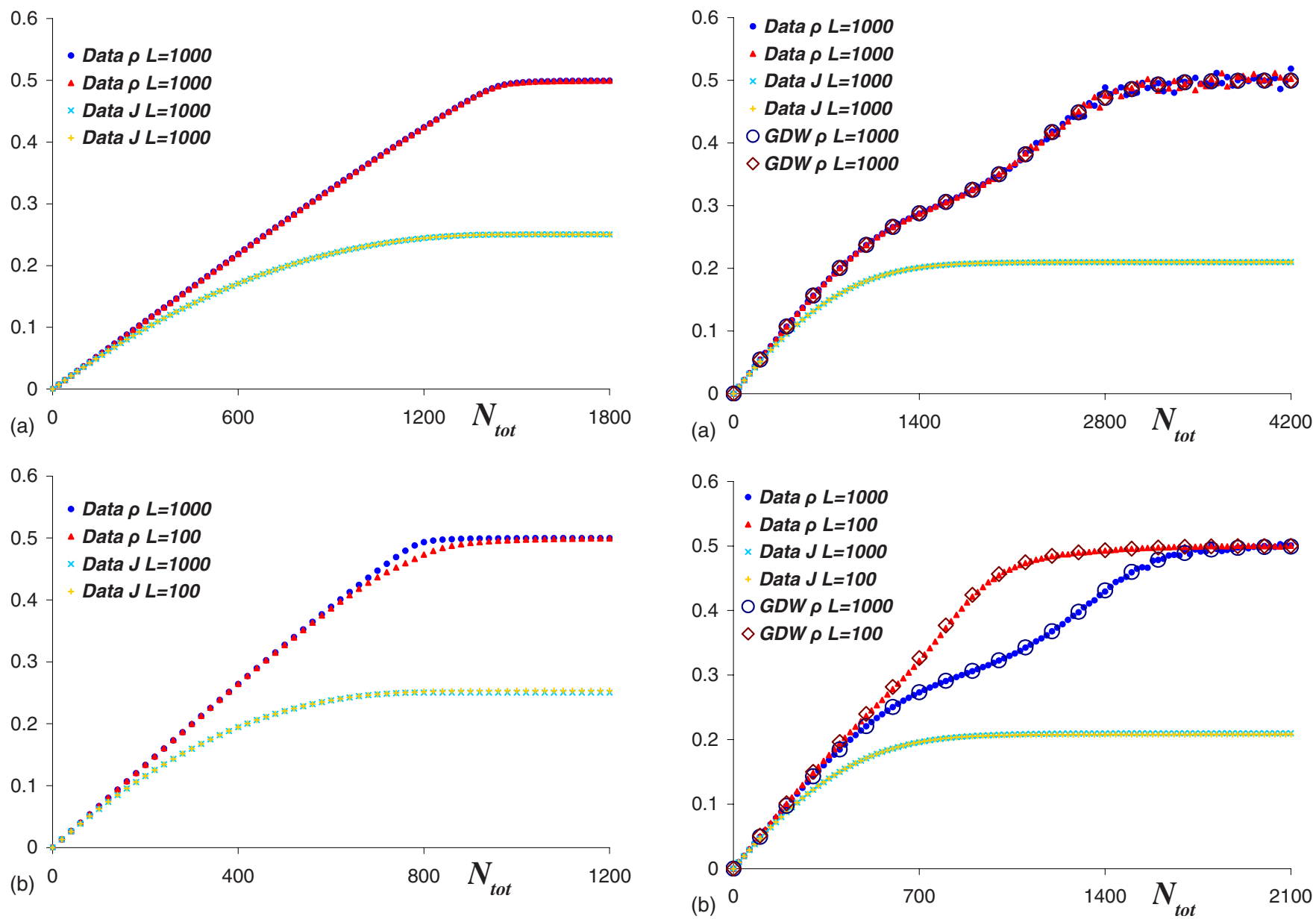

FIG. 2. (Color online) Average overall density and current as a function of $N_{t o t}$ for (a) $L_{1}=L_{2}=1000$ and (b) $L_{1}=1000$ and $L_{2}$ $=100$ with $\alpha=\beta=0.7$.

densities and currents of two competing chains behave much like those for a single TASEP, including the finite-size effects.

\section{SP}

The SP case is the most challenging due to the large fluctuations that characterize this "phase." In the standard TASEP, the system exhibits a freely moving shock separating a low-density from a high-density region. In the constrained TASEP, the crossover from the LD to the SP phase is highly nontrivial. The details depend on both the length of the chain and the functional form of $\alpha_{\text {eff }}$. Fortunately, the essentials are well captured by DW theory $[14,15,18]$ especially when it is generalized appropriately [20]. Not surprisingly, two TASEPs of equal length exhibit the same densities and currents as a function of $N_{t o t}$. Differences emerge only when $L_{1} \neq L_{2}$, as illustrated in Fig. 3, where $\alpha=\beta=0.3$ and $L_{1}$ $=1000, L_{2}=100$. These differences may be expected, however, if we recall that single TASEPs with various lengths behave quite differently when coupled to a finite pool [18]. Details of the origins of these differences in our case of two competing TASEPs can be understood in terms of the generalized DW theory [20] which we discuss in the next section. Again, like the cases above, when finite-size effects are taken

FIG. 3. (Color online) Average overall density and current as a function of $N_{t o t}$ for (a) $L_{1}=L_{2}=1000$ and (b) $L_{1}=1000$ and $L_{2}$ $=100$ with $\alpha=\beta=0.3$.

into account, there are no qualitatively new phenomena when an additional chain is included in the competition for a finite pool of particles.

\section{HD phase}

The most interesting results are observed with $\alpha$ and $\beta$ set in the HD phase. For a single constrained TASEP, there are three distinct regimes in the average total density [18], as $N_{\text {tot }}$ is varied. The behaviors for small and large $N_{t o t}$ are expected: for the former, they follow the LD behavior discussed above and for the latter, the density and current approach their asymptotic values. The presence of the third regime, interpolating between these two limits, was a surprise initially. Here, the density rises linearly with $N_{\text {tot }}$ while the current remains constant at its asymptotic limit. The origin of this linear dependence can be traced to $N_{p}$ (the reservoir occupation) being essentially constant over a range of $N_{t o t}$, so that any changes in $N_{t o t}$ are absorbed by the lattice. In particular, $N_{p}$ takes the critical value $N_{p}^{c}$, given by $\alpha_{\text {eff }}\left(N_{p}^{c}\right)$ $=\beta$, so that coexistence of low- and high-density regions on the lattice is possible. Unlike the ordinary TASEP, however, the shock does not wander over the entire lattice. Instead, it is localized at some point determined by $N_{t o t}$ with an intrin- 


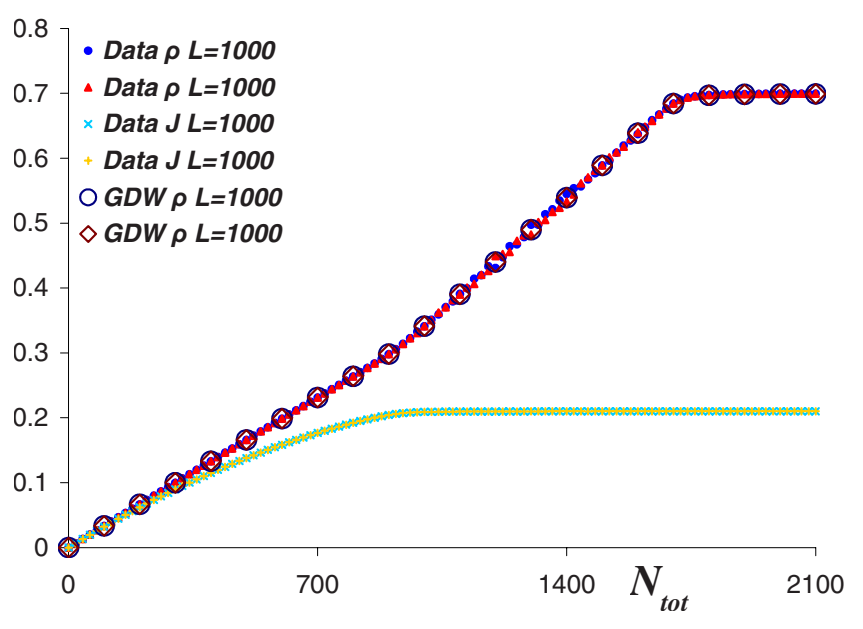

FIG. 4. (Color online) Average overall density and current as a function of $N_{t o t}$ for $L_{1}=L_{2}=1000$ with $\alpha=0.7$ and $\beta=0.3$.

sic width controlled by $\partial_{N_{p}} f[20]$. As for the current, it has already reached $\beta(1-\beta)$ at the lower extreme of this linear regime and so, it is not sensitive to the final crossover into the asymptotic HD regime.

Turning to a system with two TASEPs, there appear to be no new surprises if they are of equal lengths, as displayed in Fig. 4 for $L_{1}=L_{2}=1000$. The densities and currents for both TASEPs coincide and exhibit the three distinct regimes found in the single TASEP case. In stark contrast, however, a new feature emerges if $L_{1} \gg L_{2}$. To be specific, we will discuss the case $L_{1}=1000, L_{2}=100$ here, unless otherwise stated. While the density of the longer TASEP still displays the expected three regimes, the shorter system now develops five regimes as illustrated in Fig. 5. The two outermost regimes (for $N_{t o t}$ near zero and $N_{t o t} \rightarrow \infty$ ) are the familiar LD and asymptotic regime. In the central region, however, we see a horizontal plateau, bordered by two "crossover" regimes where the density increases steeply with $N_{t o t}$. The currents show no discernible signature whatsoever, exhibiting only the LD and the saturation regime. To appreciate the different behaviors in intuitive terms, we will present several perspectives here. In the next section, we will provide the

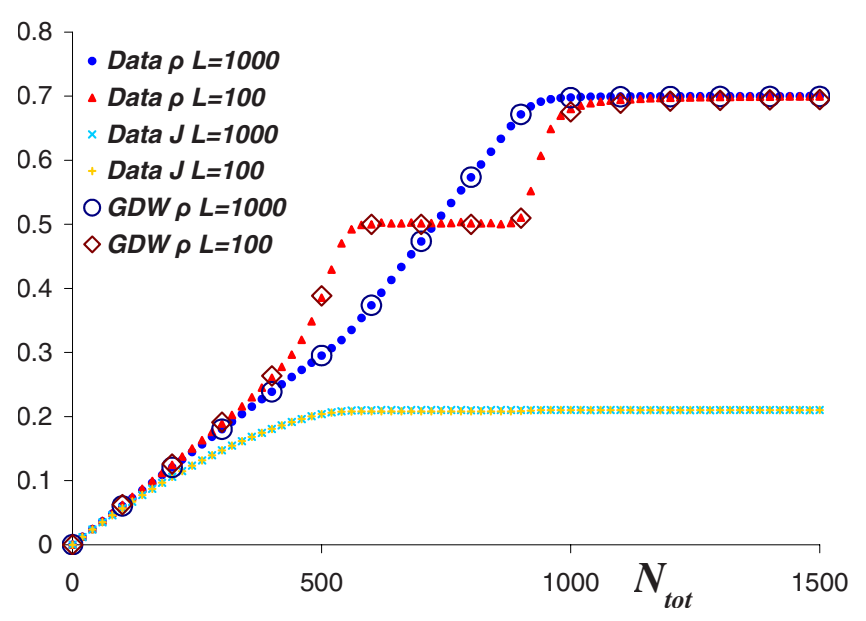

FIG. 5. (Color online) Average overall density and current as a function of $N_{t o t}$ for $L_{1}=1000$ and $L_{2}=100$ with $\alpha=0.7$ and $\beta=0.3$. mathematical approach, in which an exact solution of the generalized DW theory appears to account for all data quite well.

First, let us examine the "plateau" region. Similar to the case of a single constrained TASEP, this region is characterized by $N_{p}$ being fixed at $N_{p}^{c}$, and so, $\alpha_{e f f}=\beta$ while changes of $N_{t o t}$ are absorbed by the two TASEPs. The main difference here is that the excess particles are free to choose either chain. Given that the pool suffers only minor fluctuations, $N_{1}+N_{2}$ is also relatively constant, so that the two chains simply trade particles back and forth. In terms of the domainwall picture, a DW is present in each lattice, but their walks are completely anticorrelated. Though the shocks are no longer localized as before [20] and free to wander about, they are limited by $\left(L_{1}, L_{2}\right)$. Specifically, the shorter TASEP imposes the extent by which the DW may wander on the longer lattice. In the plateau region, the DW is free to be anywhere on the shorter lattice, so that the average profile there is strictly linear. The overall density here is strictly $1 / 2$ and the plateau emerges. In this region, all changes of $N_{t o t}$ are absorbed entirely by the longer TASEP (i.e., $\partial \rho_{1} / \partial N_{\text {tot }}$ is precisely $1 / L_{1}$ in Fig. 5). However, as will be shown below, the similarity of this behavior to the single TASEP case is deceptive. The density profiles are quite distinct reflecting the different origins of the constraints on the DW walks. This picture also shows clearly why such a plateau cannot exist for the $L_{1}=L_{2}=L$ case: neither TASEP imposes a limit on the other. By symmetry, the only point when a DW on one lattice can wander over one entire lattice is also the point where its counterpart can wander over all of the other lattice. At this point, both profiles must be linear and both densities are 1/2, so that $N_{1}+N_{2}=L$. Since the pool occupation must remain at $N_{p}^{c}$, this single point corresponds necessarily to $N_{t o t}=N_{p}^{c}+L$.

Another perspective on the existence of the plateau is offered in Fig. 6, which shows schematic views of the domains in the $N_{1}-N_{2}$ plane in which we are likely to find the $L_{1}$ $=L_{2}=1000$ system [Fig. 6(a)] and the $L_{1}=1000, L_{2}=100$ case [Fig. 6(b)]. For very small $N_{t o t}$, both TASEPs will be in the LD phase represented by the circles (green online) near the origin. As $N_{\text {tot }}$ increases beyond this regime, the system will find itself mostly in the long ellipses (red online) aligned along lines of constant $N_{1}+N_{2}$. Finally, for large $N_{t o t}$, both lattices will saturate in the HD phase indicated by the circle furthest from the origin (blue online). These two simple sketches bring out the main difference between the two cases: if the lattices are of unequal length, there is a range of $N_{\text {tot }}$ for which the size of the ellipse remains the same. In this range, the average density on the shorter TASEP is about $1 / 2$ (i.e., $N_{2} \cong 100 / 2$ here) while the occupation on the longer lattice continues to increase. When the data in Fig. 5 are replotted in the $N_{1}-N_{2}$ plane, this picture is largely confirmed: Fig. 7. In contrast, for a system with $L_{1}=L_{2}$, there is no such comparable range and so, there is no plateau region. In Fig. 8, we provide two examples of Monte Carlo data associated with these sketches. They are histograms for finding $N_{1}$ and $N_{2}$ particles in the two TASEPs. The "ridges" in these plots correspond to two of the ellipses in Fig. 6: (a) along $N_{1}+N_{2} \cong 1100$ for the $L_{1}=L_{2}=1000$ case and (b) along $N_{1}+N_{2} \cong 525$ for the other case. These ridges also indicate that, subjected to $N_{1}+N_{2} \cong$ const, all $\left(N_{1}, N_{2}\right)$ pairs are 


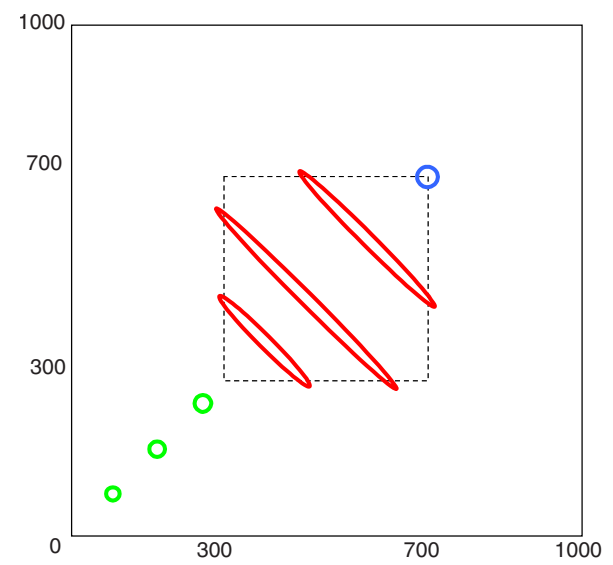

(a)

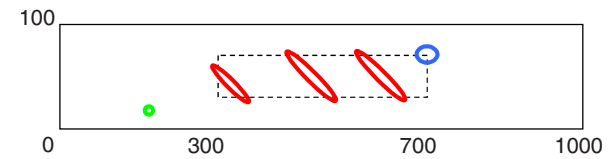

(b)

FIG. 6. (Color online) Rough sketches of distributions in the plane for (a) $L_{1}=L_{2}=1000$ and (b) $L_{1}=1000$ and $L_{2}=100$. The dashed boxes correspond to the region allowed by LD/HD coexistence (i.e., densities of $0.3 / 0.7$ here).

equally likely. Of course, these observations reflect just the picture presented above: the DWs on each chain are free to wander, but strongly anticorrelated and limited by $\left(L_{1}, L_{2}\right)$.

To provide yet another perspective, we present the density profiles of both chains in the $L_{1}=1000, L_{2}=100$ case in Fig. 9. Note that, in this figure, the horizontal axis is the fractional distance $(i / L)$ along a chain and so, points on the two curves correspond to different absolute distances $(i)$. Being linear, the profile of the short TASEP clearly reflects a totally delocalized DW. For the long chain, though the profile indicates a localized shock, this appearance is deceptive. The width (over which the profile changes from LD to HD) is actually somewhat larger than its counterpart in a single constrained TASEP (see, e.g., Fig. 2 in Ref. [20]). The origin of this broadening can be traced to the additional fluctuations allowed by the shorter chain (approximately 100 lattice sites in this case). In particular, we reexamined a single TASEP of 1000 sites at the same $\alpha$ and $\beta$ and obtained its profile. To accentuate the "interface" of the profile, we plot in Fig. 10(a) the local slopes of the profiles (i.e., $\Delta \rho(i) \equiv \rho(i+1)-\rho(i)$, shown as open diamonds). Note that these can be regarded roughly as the probability of finding the shock at site $i$. Next, we construct a "smeared" data set $\Delta \widetilde{\rho}(i \mid m)$ as follows: shift the raw data by $j=1, \ldots, m$ sites and then average over these

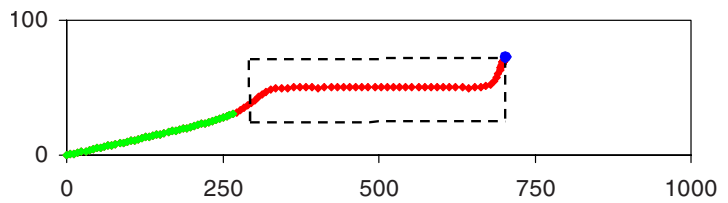

FIG. 7. (Color online) Simulation data for $L_{1}=1000$, and $L_{2}$ $=100$ with $\alpha=0.7$ and $\beta=0.3$ in the $N_{1}-N_{2}$ plane. The dashed boxes correspond to the region allowed by LD/HD coexistence.

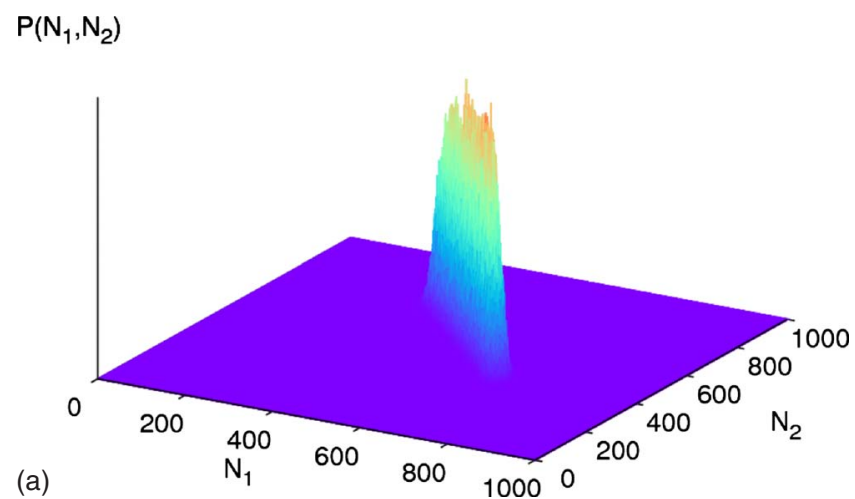

$P\left(N_{1}, N_{2}\right)$

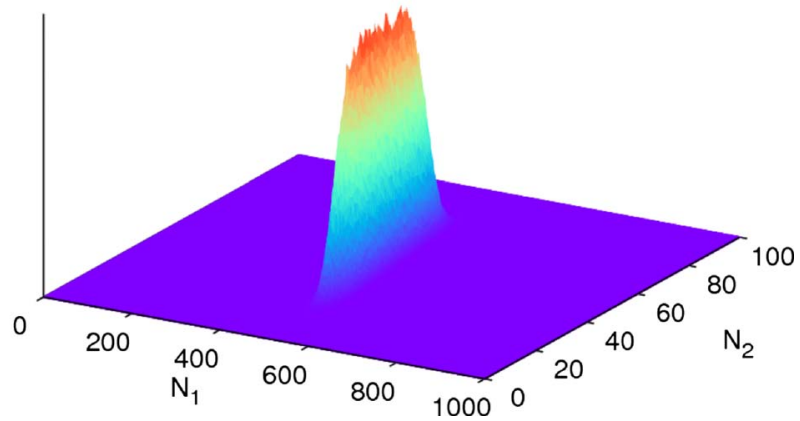

FIG. 8. (Color online) Distribution of $N_{1}$ and $N_{2}$ for (a) $N_{t o t}$ $=1400$ and $L_{1}=L_{2}=1000$ and (b) $N_{t o t}=700, L_{1}=1000$, and $L_{2}$ $=100$ with $\alpha=0.7$, and $\beta=0.3$. The $z$-axis scale for $P\left(N_{1}, N_{2}\right)$ is arbitrary in both figures.

$m$ data sets [21]. By this procedure, we hope to account for the extra degree of freedom which the shock experiences, thanks to the presence of the short TASEP. The lines (solid red, thin dashed green, and short dashed blue) in Fig. 10(a) illustrate the result of smearing with $m=100,200$, and 300 . Now we return to the two TASEP system and, in Figs. 10(b) and $10\left(\mathrm{c}\right.$ ), display $\Delta \rho(i)$ (solid squares) for the $L_{1}, L_{2}$ $=1000,100$ and 1000,300 cases, respectively. Meanwhile, the lines here (solid red and short dashed blue, respectively) are precisely those from the smeared profiles in Fig. 10(a). The good quantitative agreement between the data and these $\Delta \widetilde{\rho}(i \mid m)$ is a convincing confirmation of the picture we presented: by freely exchanging particles between the two

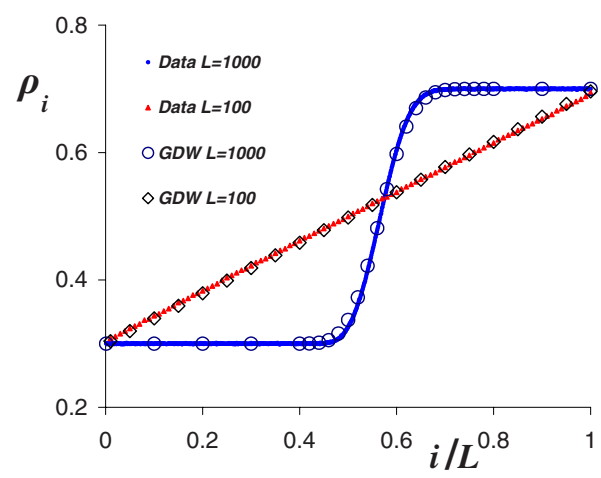

FIG. 9. (Color online) Average density profile for $N_{t o t}=700$, $L_{1}=1000$, and $L_{2}=100$ with $\alpha=0.7$ and $\beta=0.3$. 

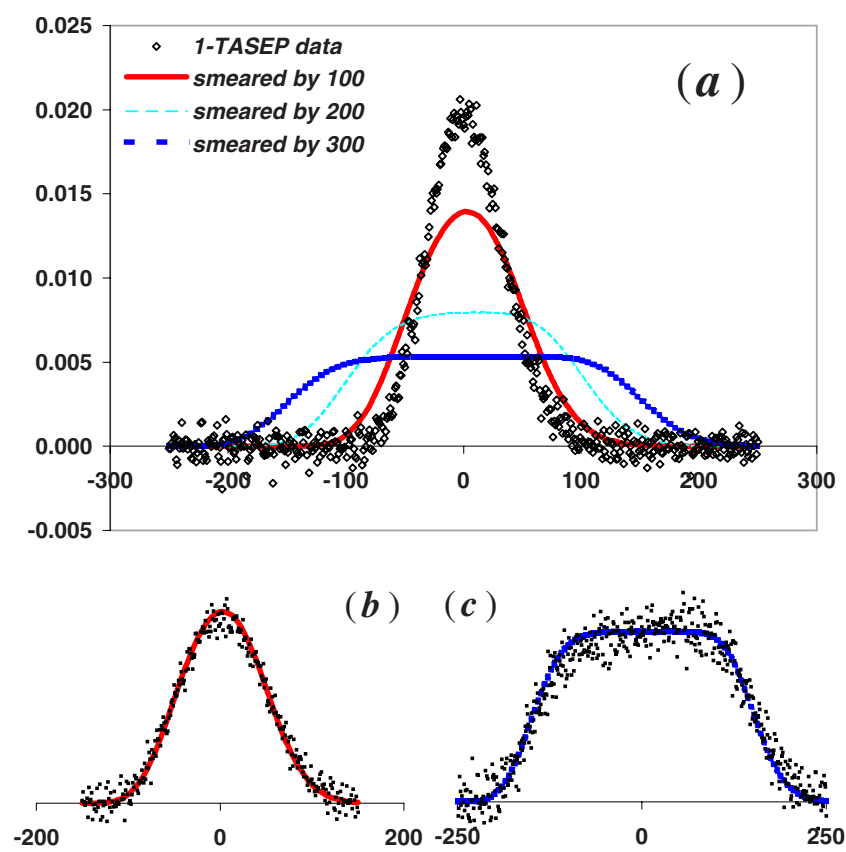

FIG. 10. (Color online) (a) Local slopes of the profile for a single TASEP with $L=1000$ and its smeared versions shown here as lines (solid red, thin dashed green, and short dashed blue). Open diamonds $(\diamond)$ are data points, obtained with $\alpha=0.7, \beta=0.3, N^{*}$ $=385, N_{\text {tot }}=650$, and shifted so that the peak lies at the origin. (b), (c) Solid squares ( $\boldsymbol{\square})$ are data points for the $L=1000$ TASEP competing with a shorter one: $L=100$ (b) and 300 (c), respectively. Lines are same as those in (a).

chains, the shorter TASEP provides extra room for an otherwise "localized" DW to wander in the longer chain. Our conclusion here is that there are two contributors to the localization of the DW in a TASEP competing for finite resources. They are (i) the feedback [20] due to a nontrivial $\alpha_{e f f}$, producing an "intrinsic" localization length, and (ii) the constraint from the other TASEP participating in the giveand-take of particles. Clearly, (ii) means that the longer chain imposes no constraint on the shorter one, so that the shock is completely delocalized regardless of mechanism (i). By the same token, the profile of the longer TASEP is typically somewhat more complex, as both mechanisms play a role. Naturally, if the chains are of equal length, delocalization prevails on both and the profiles will be linear (to the extent allowed by $N_{t o t}$ ), as our simulations confirm. Now, despite the effects of competition, it is possible to isolate the role of mechanism (i) and observe an intrinsic profile, as follows. For each measurement of $\left\{n_{\ell}(i)\right\}$, we use the totals $N_{1,2}$ to estimate the position of the DWs in each lattice: $k_{\ell}$. Then, we average the shifted occupations $\left\{n_{\ell}\left(i-k_{\ell}\right)\right\}$ to arrive at the intrinsic profile. The result is statistically identical to the profile in the single TASEP case [20], so that we are confident of the merits of the intuitive picture presented here. Details of this procedure and the comparisons will be provided elsewhere [22].

Having addressed the central plateau regime, we turn to the two bordering crossover regions. In Fig. 5, we see that the behaviors displayed here are quite rich. To highlight these better, we plot the gradients of the three occupations,

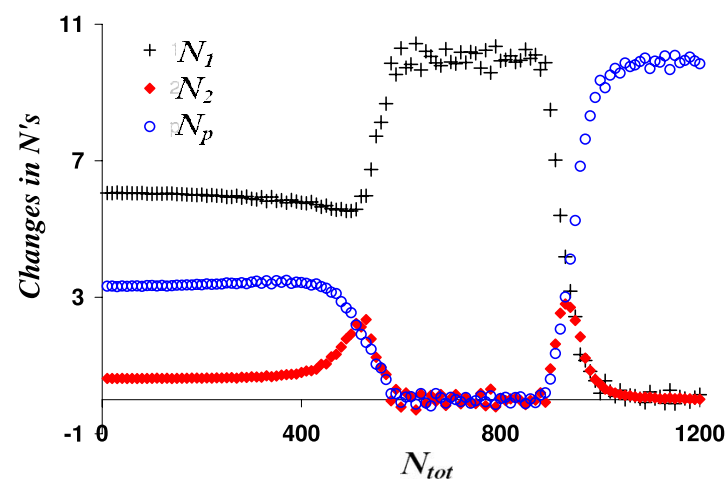

FIG. 11. (Color online) Occupation gradients of $N_{1}, N_{2}$, and $N_{p}$ with respect to $N_{\text {tot }}$ for $L_{1}=1000$, and $L_{2}=100$ with $\alpha=0.7$ and $\beta$ $=0.3$.

$N_{1,2, p}$, associated with increasing $N_{\text {tot }}$ by ten particles. In Fig. 11 , we show the data from the more interesting $L_{1}=1000$, $L_{2}=100$ case. The regions corresponding to the central plateau and its borders are most clearly seen for the small chain $\left(N_{2}\right.$, solid diamonds, red online): two peaks with a flat valley in between. At a very naive level, these features can be roughly understood from the sketch in Fig. 6(b). For small $N_{t o t}$, the circles (green online) traverse along a line with slope 1/10 corresponding to equal changes in the densities of the two chains. As $N_{t o t}$ increases further, we see an ellipse (small, red online) moving into the rectangle. Here, we might expect the two $N$ 's to increase together until the ellipse spans the vertical range of the rectangle. From there on, $N_{2}$ ceases to change while $N_{1}$ continues to increase. A similar crossover region is present for the right end of the rectangle, when $\mathrm{N}_{2}$ again increases. Though this intuitive reasoning provides a qualitatively picture of the five regimes, it clearly fails to capture the details of the two crossover regions. For example, the changes in the two N's in the first crossover are anticorrelated rather than increasing together. Evidently, these details are sufficiently subtle that they can only be fully understood in a quantitative theory for domain-wall motion-the subject of Sec. IV.

\section{E. Competition between three TASEPs}

Turning next to the study of competition between three TASEPs, we see immediately that even more scenarios are possible, from all lengths being equal, to some being the same, to all lengths being drastically different. Though we have explored quite a few cases, we will only present data for the most extreme one (HD): $L_{1}=1000, L_{2}=100$, and $L_{3}$ $=10$ with $\alpha=0.7$ and $\beta=0.3$. How the three densities vary as we increase $N_{\text {tot }}$ is shown in Fig. 12. We again see the smaller two TASEPs entering into a plateau regime, with linear profiles and half filling on the average. Meanwhile, the longest chain again displays a localized shock as shown in Fig. 13. In more detail, the shortest TASEP reaches $\rho=1 / 2$ first, followed by the intermediate chain. The behavior of the longest TASEP is much the same as what we observed in the $L=1000$ chain above. It appears that adding a third chain does not lead to any qualitatively novel behavior. The overall currents, especially if the severe finite-size effects associated 


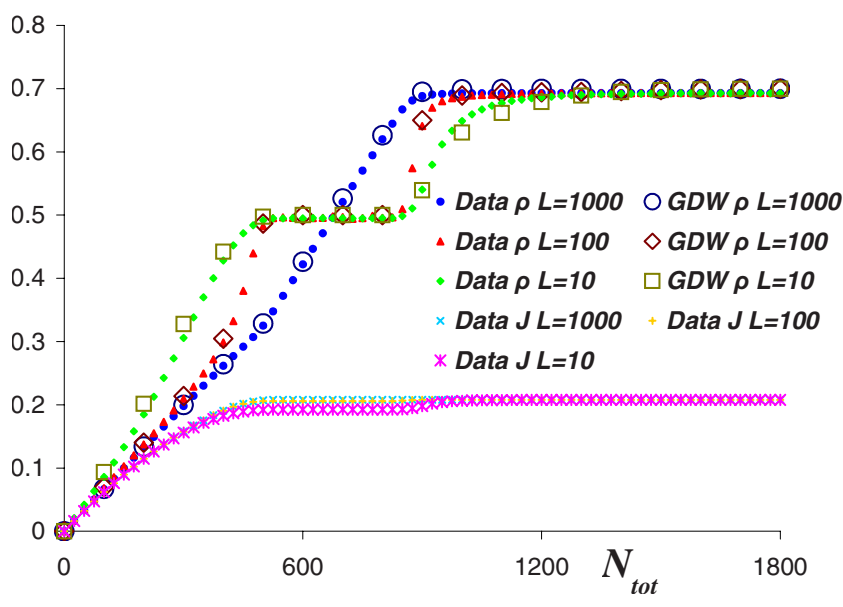

FIG. 12. (Color online) Average overall density and current as a function of $N_{t o t}$ for $L_{1}=1000, L_{2}=100$, and $L_{3}=10$ with $\alpha=0.7$ and $\beta=0.3$.

with $L=10$ are taken in account, also display few surprises. Of course, the phase space for $M=3$ is considerably larger and phenomena may very well emerge upon closer examination. Further studies are in progress and will be reported elsewhere. In the remainder of this paper, we will focus on a quantitatively viable analytic picture for an arbitrary number of TASEPS.

\section{GENERALIZED DOMAIN-WALL THEORY}

To understand most of the phenomena we observed, it is sufficient to use the simplest approximation [18], based on self-consistent equations between the feedback dependence, $\alpha_{e f f}\left(N_{p}\right)$, and the occupation variables, $N_{\ell}$, for the TASEPs. The only serious complication arises in the HD case, when $\alpha_{\text {eff }}$ reaches $\beta$ and each individual TASEP enters an SP-like regime. As presented above, a variety of interesting behaviors emerge for which intuitively reasonable simple arguments paint a good qualitative picture. In this section, we will provide a quantitative description, which relies on an excellent approximation, namely, an appropriately generalized domain-wall theory $[18,20]$. Proposed about a decade ago for the standard single TASEP $[14,15]$, DW theory assumes that the configurations are well accounted for by those

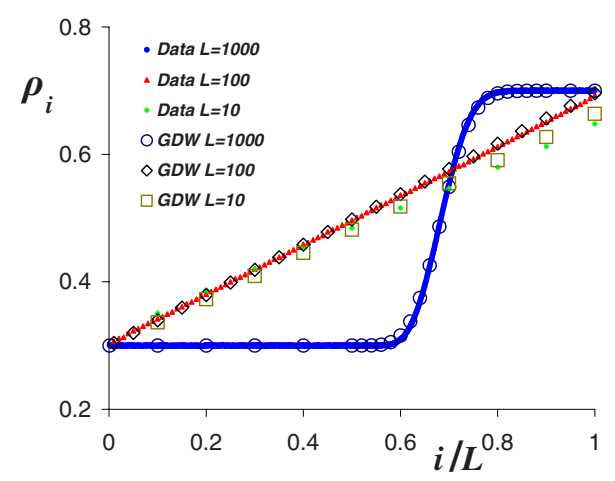

FIG. 13. (Color online) Average density profile for $N_{\text {tot }}=600$, $L_{1}=1000, L_{2}=100, L_{1}=10$ with $\alpha=0.7$ and $\beta=0.3$. with a microscopic interface between two regions, one with high density $\rho_{+}$and another with low density $\rho_{-}$. The generalization to a single TASEP constrained by finite resources $[18,20]$ provided excellent agreement with all aspects of simulation data. Referring the reader to [20] for details, let us first present a brief summary of this approach here in the context of multiple TASEPs competing for the same pool of particles.

We assume that a configuration of the system of $M$ TASEPs can be well approximated by specifying the position of the shocks (DWs) on each lattice, $k_{\ell} \in\left[0, L_{\ell}\right]$, with $\ell$ $=1, \ldots, M$. Since all chains are subjected to the same entry and exit rates of particles, we will further assume that the densities before (sites $i \leq k$ ) and after (sites $i>k$ ) the wall on all chains are identical, i.e., $\rho_{-}=\alpha_{\text {eff }}$ and $\rho_{+}=1-\beta$, respectively. Now, $\alpha_{e f f}$ depends on the numbers in the pool and so, on the occupation on the lattices, $N_{\ell}$. Thus, to close the equations, we need the relationship between $N_{\ell}$ and $k_{\ell}$ :

$$
\begin{gathered}
N_{\ell}=\rho_{-} k_{\ell}+\rho_{+}\left(L_{\ell}-k_{\ell}\right) \\
=\left(\alpha_{e f f}+\beta-1\right) k_{\ell}+(1-\beta) L_{\ell},
\end{gathered}
$$

which leads us to the pool occupation,

$$
N_{p}=N_{t o t}-\sum_{\ell} N_{\ell}
$$

and the dependence of $\alpha_{e f f}$ on the $k$ 's. In short, from $\alpha_{e f f}$ $=\alpha f\left(N_{p}\right)=\alpha \tanh \left(\left[N_{t o t}-\Sigma_{\ell} N_{\ell}\right] N^{*}\right)$, we have

$$
\alpha_{e f f}(K)=\alpha \tanh \left(\frac{N_{t o t}-(1-\beta) \sum_{\ell} L_{\ell}-\left(\alpha_{e f f}+\beta-1\right) \sum_{\ell} k_{\ell}}{N *}\right) .
$$

Note that $\alpha_{\text {eff }}$ depends only on the sum

$$
K \equiv \sum_{\ell=1}^{M} k_{\ell}
$$

rather than the individual $k$ 's. This simplification will be crucial for us to find an exact steady-state solution to the master equation in our system. Of course, we must solve the nonlinear, self-consistent Eq. (11) to determine $\alpha_{\text {eff }}(K)$. This task was performed numerically as in the previous study [20]. Indeed, we have the same functional form as before, except that we now encounter $\alpha_{e f f}\left(K ; \Sigma_{\ell} L_{\ell}\right)$ instead of $\alpha_{e f f}(k ; L)$.

Once $\alpha_{e f f}(K)$ is known, the rates for the DW to hop to the left $\left(D^{-}\right)$and right $\left(D^{+}\right)$can be computed. We denote explicitly their $K$ dependence by a subscript:

$$
\begin{gathered}
D_{K}^{-}=\frac{\alpha_{e f f}\left(1-\alpha_{e f f}\right)}{1-\beta-\alpha_{e f f}}, \\
D_{K}^{+}=\frac{\beta(1-\beta)}{1-\beta-\alpha_{e f f}} .
\end{gathered}
$$

With these, the master equation for $P\left(\left\{k_{\ell}\right\}, t\right)$, the probability to find DWs at $\left\{k_{\ell}\right\}$ at time $t$ is well defined. For $\left\{k_{\ell}\right\}$ lying in the interior of the allowed domain, it is easy to write 


$$
\begin{aligned}
\partial_{t} P\left(\left\{k_{\ell}\right\}, t\right)= & \sum_{j=1}^{M}\left[D_{K+1}^{-} P\left(\left\{k_{\ell}+\delta_{\ell j}\right\}, t\right)+D_{K-1}^{+} P\left(\left\{k_{\ell}-\delta_{\ell j}\right\}, t\right)\right] \\
& -M\left[D_{K}^{-}+D_{K}^{+}\right] P\left(\left\{k_{\ell}\right\}, t\right),
\end{aligned}
$$

where $\delta_{\ell j}$ is the Kronecker delta. At the boundaries, we must impose reflecting boundary conditions on the appropriate $k$ 's. Since there are quite a few $\left(3^{M}-1\right)$ of these conditions, it will be helpful to begin by studying the $M=2$ case explicitly (four corners and four sides of a rectangle). In the next subsection, we will provide some details for obtaining the steady-state solution associated with this case. We will find that the natural variables are $\left(K, Q \equiv k_{1}-k_{2}\right)$ rather than $\left(k_{1}, k_{2}\right)$. The insights gained here will facilitate the analysis of the arbitrary $M$ case to be presented in the last subsection.

\section{A. Case with two TASEPs}

Here, we focus on the steady-state solution to Eq. (16) for $M=2$, for $0<k_{1}<L_{1}$ and $0<k_{2}<L_{2}$. Dropping the $t$, and setting the left to zero, this equation reduces to

$$
\begin{aligned}
0= & D_{K+1}^{-}\left[P\left(k_{1}+1, k_{2}\right)+P\left(k_{1}, k_{2}+1\right)\right]+D_{K-1}^{+}\left[P\left(k_{1}-1, k_{2}\right)\right. \\
& \left.+P\left(k_{1}, k_{2}-1\right)\right]-2\left[D_{K}^{-}+D_{K}^{+}\right] P\left(k_{1}, k_{2}\right),
\end{aligned}
$$

where

$$
K=k_{1}+k_{2}
$$

here. The minimum boundary conditions to be imposed correspond to one or both DWs being reflected from the ends of the lattices. Thus, at the four sides of the rectangle, we write

$$
\begin{aligned}
0= & D_{k_{1}+1}^{-}\left[P\left(k_{1}+1,0\right)+P\left(k_{1}, 1\right)\right]+D_{k_{1}-1}^{+} P\left(k_{1}-1,0\right) \\
& -\left[D_{k_{1}}^{-}+2 D_{k_{1}}^{+}\right] P\left(k_{1}, 0\right), \\
0= & D_{k_{2}+1}^{-}\left[P\left(0, k_{2}+1\right)+P\left(1, k_{2}\right)\right]+D_{k_{2}-1}^{+} P\left(0, k_{2}-1\right) \\
& -\left[D_{k_{2}}^{-}+2 D_{k_{2}}^{+}\right] P\left(0, k_{2}\right), \\
0= & D_{L_{2}+k_{1}-1}^{+}\left[P\left(k_{1}-1, L_{2}\right)+P\left(k_{1}, L_{2}-1\right)\right] \\
+ & D_{L_{2}+k_{1}+1}^{-} P\left(k_{1}+1, L_{2}\right)-\left[D_{L_{2}+k_{1}}^{+}+2 D_{L_{2}+k_{1}}^{-}\right] P\left(k_{1}, L_{2}\right), \\
0 & D_{L_{1}+k_{2}-1}^{+}\left[P\left(L_{1}, k_{2}-1\right)+P\left(L_{1}-1, k_{2}\right)\right] \\
+ & D_{L_{1}+k_{2}+1}^{-} P\left(L_{1}, k_{2}+1\right)-\left[D_{L_{1}+k_{2}}^{+}+2 D_{L_{1}+k_{2}}^{-}\right] P\left(L_{1}, k_{2}\right) .
\end{aligned}
$$

Similarly, the conditions at the four corners are

$$
\begin{gathered}
0=D_{1}^{-}[P(0,1)+P(1,0)]-2 D_{0}^{+} P(0,0), \\
0=D_{L_{1}+L_{2}-1}^{+}\left[P\left(L_{1}-1, L_{2}\right)+P\left(L_{1}, L_{2}-1\right)\right] \\
-2 D_{L_{1}+L_{2}}^{-} P\left(L_{1}, L_{2}\right), \\
0=D_{L_{1}-1}^{+} P\left(L_{1}-1,0\right)+D_{L_{1}+1}^{-} P\left(L_{1}, 1\right)-\left[D_{L_{1}}^{+}+D_{L_{1}}^{-}\right] P\left(L_{1}, 0\right),
\end{gathered}
$$

$$
0=D_{L_{2}-1}^{+} P\left(0, L_{2}-1\right)+D_{L_{2}+1}^{-} P\left(1, L_{2}\right)-\left[D_{L_{2}}^{+}+D_{L_{2}}^{-}\right] P\left(0, L_{2}\right) .
$$

However, as discovered in a previous study [20], there is a more subtle boundary condition. For sufficiently low $N_{t o t}$, it is not possible for one or both DWs to reach the left boundary. Specifically, if $N_{t o t} \leq(1-\beta)\left(L_{1}+L_{2}\right)$, then at least one of the lattices cannot be filled with the high density, $\rho_{+}$, so that the sum of the DW positions must be larger than $K_{\min } \equiv L_{1}$ $+L_{2}-N_{t o t} /(1-\beta)$. Another way to understand this limit is that the pool is empty $\left(N_{p}=0\right)$ when $K$ reaches $K_{\min }$. Both $\alpha_{e f f}$ and $D^{-}$vanish and we simply have $P \equiv 0$ for $k_{1}+k_{2}$ $\leq K_{\min }$. To summarize, we see that $P\left(k_{1}, k_{2}\right)$ can be nonzero only in a (generally) "cut-rectangular" domain:

$$
\mathcal{R}: 0 \leq k_{1} \leq L_{1}, \quad 0 \leq k_{2} \leq L_{2}, \quad K_{\min }<k_{1}+k_{2} .
$$

Taking into account this complication of the boundary conditions and thanks to $D$ being dependent on only one variable, these equations can be solved analytically. In particular, although the original competing TASEPs is a nonequilibrium statistical mechanics problem, the DW approximation reduces it to the point that detailed balance prevails. Specifically, the product of the rates around every elementary loop [through the four points $\left(k_{1}, k_{2}\right) ;\left(k_{1}+1, k_{2}\right) ;\left(k_{1}+1, k_{2}\right.$ $\left.+1) ;\left(k_{1}, k_{2}+1\right)\right]$ in configuration space is

$$
D_{K}^{+} D_{K+1}^{+} D_{K+2}^{-} D_{K+1}^{-}
$$

regardless of the direction taken around the loop. Since all closed loops in this space are composed of these elementary loops, the Kolmogorov criterion [23] is satisfied. Thus, we have detailed balance

$$
D_{K}^{-} P\left(k_{1}, k_{2}\right)=D_{K-1}^{+} P\left(k_{1}-1, k_{2}\right)=D_{K-1}^{+} P\left(k_{1}, k_{2}-1\right)
$$

and the solution can be obtained by recursion. Setting

$$
P\left(L_{1}, L_{2}\right) \equiv Z^{-1}
$$

where $Z$ is a (normalization) constant, we easily find the stationary distribution, for $K \leq L_{1}+L_{2}-1$,

$$
P\left(k_{1}, k_{2}\right)=Z^{-1} \Phi(K),
$$

provided $\left(k_{1}, k_{2}\right)$ lies in $\mathcal{R}$. Here, we define

$$
\Phi(K) \equiv \prod_{j=K}^{L_{1}+L_{2}-1} \frac{D_{j+1}^{-}}{D_{j}^{+}}
$$

and emphasize that $P\left(k_{1}, k_{2}\right)$ varies only via the sum $k_{1}+k_{2}$ and is flat along lines of constant $K$. Due to the irregular shape of $\mathcal{R}$, the normalization factor $Z$ is straightforward to find but not simply $\Sigma_{K} \Phi(K)$, as in the single TASEP case [20]. For example, if $0<K_{\min }<L_{1}$, then

$$
Z=1+\sum_{K=K_{\min }+1}^{L_{1}+L_{2}-1}\left[\left(L_{1}-K\right) \Theta\left(L_{1}-K\right)+L_{2}+1\right] \Phi(K),
$$

where $\Theta$ is the Heaviside function. 
Since this stationary distribution depends only on the sum of the shock positions, it behooves us to change the variables from $\left(k_{1}, k_{2}\right)$ to $(K, Q)$, where

$$
Q \equiv k_{1}-k_{2} \text {. }
$$

In terms of these variables, Eq. (16) becomes

$$
\begin{aligned}
0= & D_{K+1}^{-}[P(K+1, Q+1)+P(K+1, Q-1)] \\
& +D_{K-1}^{+}[P(K-1, Q-1)+P(K-1, Q+1)] \\
& -2\left[D_{K}^{+}+D_{K}^{-}\right] P(K, Q)
\end{aligned}
$$

with similar replacements for the eight boundary conditions. The detailed balance condition, Eq. (28), now reads

$$
D_{+K}^{+} P(K, Q)=D_{K+1}^{-} P(K+1, Q+1)=D_{K+1}^{-} P(K+1, Q-1)
$$

and suggests a solution that depends only on $K$. Assuming the ansatz

$$
P(K, Q)=Z^{-1} \Phi(K) H(Q, K),
$$

where $H(Q, K)$ is a Heaviside-like function (unity in $\mathcal{R}$ and zero otherwise), we find $\Phi(K+1) \propto \Phi(K) D_{K}^{+} / D_{K+1}^{-}$. Taking some care with the boundary conditions, it is easy to verify that form (36) is indeed valid.

Before turning to a theory for the multi-TASEP case, let us compare the predictions (with no adjustable parameters) of this approach with the data. First, the density profiles can be obtained, following the methods in the previous study [20]:

$$
\begin{aligned}
& \rho_{1}(i)=\sum_{k_{2}=0}^{L_{2}}\left[(1-\beta) \sum_{k_{1}=0}^{i} P\left(k_{1}, k_{2}\right)+\sum_{k_{1}=i+1}^{L_{1}} \alpha_{e f f(K)} P\left(k_{1}, k_{2}\right)\right], \\
& \rho_{2}(i)=\sum_{k_{1}=0}^{L_{1}}\left[(1-\beta) \sum_{k_{2}=0}^{i} P\left(k_{1}, k_{2}\right)+\sum_{k_{2}=i+1}^{L_{2}} \alpha_{e f f(K)} P\left(k_{1}, k_{2}\right)\right] .
\end{aligned}
$$

In this expression, it is clear that $\Sigma_{k_{2}} P\left(k_{1}, k_{2}\right)$ is just the probability for finding the DW at $k_{1}$ regardless of the configuration in the other TASEP and similarly for $\Sigma_{k_{1}} P$. We find that these agree well with all observed profiles-not only for regimes with little structure but also for complex situations such as the plateau region. As an illustration, in Fig. 9 we show profiles for the familiar $L_{1}=1000, L_{2}=100$ case with $N_{t o t}=700$. It is clear that the essentials of our system, such as a linear profile in one chain along with a localized shock in the other, have been successfully captured in this theory. From these profiles, we obtain the overall densities by $\rho_{\ell}$ $=\Sigma_{i} \rho_{\ell}(i) / L_{\ell}$. As shown in Figs. 1 and 3-5, they are in excellent agreement with the simulation data. Not surprisingly, the histograms for $\left(N_{1}, N_{2}\right)$ shown in Fig. 8 can also be predicted, being basically $P\left(k_{1}, k_{2}\right)$ via correspondence (9). Although the agreement is also quite good, we should remark on two shortcomings. First, relationship (9) between $N$ and $k$ cannot be exact, since both $N$ and $k$ are integer valued. Second, lattices with $N$ greater than $\rho_{+} L$ or less than $\rho_{-} L$ are obviously absent from the theory, a limitation due to the DW approximation. However, in all the regions we have explored, these shortcomings result only in minor disparities.

Since so many aspects of our system can be understood by this approach, let us return to form a better intuitive picture for the two crossover regions for the $L_{1}=1000, L_{2}$ $=100$ case discussed at the end of Sec. III D. First, note that there are subtle changes in the gradients of $N_{\ell}$, as $N_{\text {tot }}$ increases up to the lower crossover $\left(N_{t o t} \sim 400\right.$ in Fig. 11). By contrast, $\partial N_{p} / \partial N_{t o t}$ remains relatively constant. More significantly, the longer chain "loses" while the shorter one "wins." This behavior can be traced to the DWs being mostly bound to the exit (right edge), but making longer and longer excursions into the lattice as particles in the system become more abundant. The exponential tails of this excursion are essentially identical provided they do not intrude significantly into the entry (left edge). Indeed, if we measure the ratio of the profiles $\rho_{1}(900+i) / \rho_{2}(i)$, it is essentially unity for $N_{t o t}$ up to $\sim 400$. Nonetheless, the overall density of the shorter lattice is affected more by a similar portion of an enhanced profile (over $\rho_{-}$), and so, will increase faster. We should also remark that the density to the left of the shock (low-density region) in either lattice has yet to reach the final value (i.e., $\rho_{-}<\beta$ ).

After we enter the first crossover region $\left(N_{t o t} \sim 400\right)$, the DW wanders further from the exit in each lattice. It eventually reaches the left side of the smaller TASEP and enters the SP. To understand how this transition occurs, let us look at how the density of the ordinary unconstrained TASEP of infinite length changes as a function of $\alpha$ with $\beta<1 / 2$ as we cross the first order line $\alpha=\beta$. Starting from $\alpha=0$, the density rises linearly until $\alpha=\beta$. At this point, the density jumps to $1 / 2$. It then jumps again to a value of $1-\beta$ for $\alpha>\beta$. For a system with a finite length, these jumps are no longer sharp, but smeared out near the value of $\alpha=\beta$, reflecting a rapid increase in the density (and number of particles) in this region. We now use this information, noting that (i) $N_{t o t}$ controls the effective $\alpha$, and (ii) $\alpha_{e f f}=\beta$ spans a whole region of $N_{t o t}$, and (iii) that the shorter TASEP behaves essentially like an unconstrained one, since it can draw from both the pool and the longer chain. The first rapid increase, as $\alpha_{e f f}$ approaches $\beta$ from below, is responsible for the first peak of $d N_{2} / d N_{\text {tot }}$ just above $N_{\text {tot }}=400$ shown in Fig. 11. Clearly, the gradient then decays to zero as $N_{2}$ reaches the characteristic plateau. The next peak reflects the end of the $\alpha_{\text {eff }}=\beta$ region: it is related to the sharp increase in the density on the other side of the first order line. In terms of the domain-wall picture, the first peak reflects the fact that the exponential tail in the density profile reaches the entrance and changes from an exponential decay into a linear one. In other words, the probability of finding the shock near the entrance increases until it is flat across the whole system. At the second crossover, the reverse happens: the system is in a high-density phase, with a small exponential tail at the entrance, indicating that the shock is now predominantly found there.

It is interesting to note that, in the first crossover regime, the pool loses steadily at sharing the increases in $N_{t o t}$. Of course, over the plateau regime, only the longer chain gains from the changes in $N_{t o t}$. The picture for the second crossover regime is essentially the same, except occurring in "reverse order." Needless to say, the pool is the ultimate winner 
in this competition absorbing all increases in $N_{\text {tot }}$ beyond this regime.

\section{B. Multiple TASEPs}

The insights gained in the detailed analysis for two TASEPs greatly facilitate investigation of the general multiTASEP case here. In particular, since the entry rates into all $M$ chains are the same, $\alpha_{e f f}$, and depend only on $K=\Sigma_{\ell} k_{\ell}$, the transition probabilities in master equation (16) again satisfy the Kolmogorov criterion. A change in coordinates similar to the two TASEP case can be performed, with $K \equiv \Sigma k_{\ell}$ as the special variable. The boundary conditions are also straightforward though considerably more tedious, since the $M$-dimensional generalization of $\mathcal{R}$ is more complicated in general. Nevertheless, thanks to detailed balance, it is simple to solve for the stationary distribution associated with this (effectively one-dimensional) master equation. The answer will again be form (36):

$$
P\left(\left\{k_{\ell}\right\}\right)=Z^{-1} \Phi(K) H\left(\left\{k_{\ell}\right\}\right),
$$

where the Heaviside-like function is now defined for the $M$-dimensional $\mathcal{R}$. Needless to say, the "cuts" of constant $K$ across $\mathcal{R}$ are geometric objects of dimension $M-1$, the general shapes of which are far more complicated than the lines in the $M=2$ case above. As a result, the normalization factor is given by a much more complex expression than Eq. (32), since the coefficients involve polynomials (in $K$ ) up to order $M-1$. Nevertheless, these computations are very simple for modern computers and $P\left(\left\{k_{\ell}\right\}\right)$ can be easily accessed numerically for a reasonable range of $M$ 's. For example, we computed $P\left(k_{1}, k_{2}, k_{3}\right)$ for the three TASEP case discussed in the previous section $\left(L_{1}=10 L_{2}=100 L_{3}=1000\right.$, with $\alpha=0.7$ $=1-\beta)$. From $P$, we obtained the average profiles and overall densities as a function of $N_{\text {tot }}$. As shown in Fig. 12, there is excellent agreement between this theory and the data, over the many regimes encountered while $N_{\text {tot }}$ increases. Similarly, we find good agreement for the average density profiles as illustrated in Fig. 13 for the case with $N_{\text {tot }}=600$. While two profiles are linear and one contains a localized shock, all properties are well predicted by this approach. Of course, the crossover regimes are richer, as each of the shorter chains produces peaks in the gradients similar to those shown in Fig. 11. With no qualitatively new phenomena, these and other cases, as well as further details, will be discussed in a later publication [22]. Our conclusion is that the generalized DW theory is remarkably successful at capturing the essence of our problem. Only the study of very sensitive quantities reveals a poorer match between this approach and simulation data.

To end this section, let us note that there is a thermal analog for $P\left(\left\{k_{\ell}\right\}\right)$, namely, the canonical ensemble. Here, $K$ plays the role of the total energy, $E$, while the total number of points in a sheet of fixed $K$ corresponds to the microcanonical partition function, $\Omega(E)$. The point $\left\{k_{\ell}\right\}=\left\{L_{\ell}\right\}$ (highest $K$ allowed) would be the "ground state," while the precise connection between $E$ and $K$ is given by $E=-k_{B} T \ln \Phi(K)$. Of course, we chose to label our normalization constant $Z$ to carry this analogy to its logical end. Meanwhile, $N_{\text {tot }}$ seems to play the role of temperature, with the average of $-K$ being a monotonically increasing function of $N_{t o t}$. Exploring this correspondence will be both interesting and imperative, especially if we hope to make progress toward our goal, namely, a cell with a few thousand different genes, each appearing in hundreds of copies.

\section{SUMMARY AND OUTLOOK}

In this paper, we explored how competition between TASEPs affects the density profile, the overall density, and the current for each chain. A feedback mechanism introduced previously [18] was implemented. We used Monte Carlo simulations to explore the properties of the overall densities, currents, and profiles of the TASEPs for a variety of parameters (lattice length, $N_{t o t}, \alpha$, and $\beta$ ). The competition produced several features that are absent from the case of a single TASEP constrained by finite resources $[18,20]$. There, the feedback serves to localize a domain wall when the control parameters are set in favor of its appearance in the lattice. Here, the presence of other TASEPs adds an extra dimension to the feedback leading to the delocalization of the DW to the extent allowed by the lengths of the other chains. Thus, when a long chain competes with a short one, its DW wandering is limited by the length of the latter. By contrast, a DW in a short chain is free to roam over the entire lattice, so that the average profile displayed is strictly linear and the average overall density is just $1 / 2$. This picture can be readily generalized to three or more chains and is confirmed in limited simulation studies with three competing TASEPs.

For the single TASEP with feedback, the standard domain-wall theory was appropriately generalized and proved to be extremely successful [20]. Extending this theory to an arbitrary number of TASEPs is straightforward and a master equation for $P\left(\left\{k_{\ell}\right\}, t\right)$, where $k_{\ell}$ denotes the position of the DW in the $\ell$ th chain, is easily formulated. Fortunately, we are able to find the steady-state solution in a system where the entry rates onto all chains are the same. The details were presented for the two TASEP case and steps for extending it to arbitrary $M$ were provided. A remarkable feature is the existence of an intimate mapping from our steady state to a canonical equilibrium ensemble. From this stationary distribution, all density profiles and currents can be predicted with no adjustable parameters. The results agree well with all data and give us much insight into a variety of phenomena discovered in simulations.

The most intriguing questions beyond our study here focus on time-dependent phenomena. Even for the standard TASEP, there is a wealth of interesting dynamics $[8,13]$. How are these affected by constraints of finite resources and competition? For example, one of the simplest quantities displaying remarkable behavior in the open TASEP is the power spectrum associated with the total occupation, $N(t)$ [16]. Preliminary data for a single chain coupled to finite resources reveal a host of phenomena [24]. With many chains competing for one pool of particles, we can study many other quantities such as correlations between the various TASEPs. Hopefully, these pursuits will reveal other exciting secrets in this system and provide us with a deeper insight. 
Being motivated by protein synthesis in cells, there are many extensions we can explore. Here we list some examples. The initiation rates for various genes are far from being identical. Thus, we should introduce a full set of $\left\{\alpha_{\ell}\right\}$ s, to model highly and rarely expressed genes. Naturally, these will induce complex $\left\{k_{\ell}\right\}$ dependences in the DW hopping rates: $D^{ \pm}\left(\left\{k_{\ell}\right\}\right)$. One serious consequence is that the transition rates in master equation (16) will typically violate the Kolmogorov condition, so that the steady-state solution will be truly nonequilibrium in character. Nontrivial steady-state probability currents necessarily follow [25] and their implications surely deserve further pursuit. Other obvious extensions include important aspects of protein synthesis which have been considered in earlier models, such as having particles with finite extent to model the fact that ribosomes are relatively large molecules "covering" many codons [4] and the inclusion of inhomogeneous hopping rates along the lattice to model the inhomogeneous sequence of codons and the wide range of concentrations of their associated aa-tRNAs
[26]. Another aspect is the ribosome recycling enhancement considered by Chou [19] and the role of diffusion of (the subunits of) ribosomes in a competitive environment. Along these lines, to model the workings of a cell better, we should consider a system with some regulation on $N_{t o t}$ as opposed to being just a preassigned fixed number. Finally, an even more ambitious goal is to include not only the competition for ribosomes, but also for the many varieties of aa-tRNA molecules. Clearly, much work remains to be done in order to arrive at a realistic model of, and to better understand, protein synthesis in a cell.

\section{ACKNOWLEDGMENTS}

The authors would like to thank D. A. Adams, T. Chou, B. Derrida, Jiajia Dong, E. Levine, and G. M. Schütz for stimulating discussions. This work was supported in part by the U.S. National Science Foundation through Grant No. DMR0705152 .
[1] Committee on CMMP 2010, Solid State Sciences Committee, Condensed-Matter and Materials Physics: The Science of the World Around Us (National Research Council/National Academies Press, Washington, DC, 2007).

[2] B. Derrida, Phys. Rep. 301, 65 (1998).

[3] G. M. Schütz, in Phase Transitions and Critical Phenomena, edited by C. Domb and J. L. Lebowitz (Academic, London, 2001), Vol. 19.

[4] C. MacDonald, J. Gibbs, and A. Pipken, Biopolymers 6, 1 (1968); C. MacDonald and J. Gibbs, ibid. 7, 707 (1969); L. B. Shaw, R. K. P. Zia, and K. H. Lee, Phys. Rev. E 68, 021910 (2003); T. Chou and G. Lakatos, Phys. Rev. Lett. 93, 198101 (2004); J. J. Dong, B. Schmittmann, and R. K. P. Zia, J. Stat. Phys. 128, 21 (2007); J. J. Dong, B. Schmittmann, and R. K. P. Zia, Phys. Rev. E 76, 051113 (2007).

[5] V. Popkov, A. Rákos, R. D. Willmann, A. B. Kolomeisky, and G. M. Schütz, Phys. Rev. E 67, 066117 (2003); M. R. Evans, R. Juhász, and L. Santen, ibid. 68, 026117 (2003); R. Juhász and L. Santen, J. Phys. A 37, 3933 (2004).

[6] D. Chowdhury, L. Santen, and A. Schadschneider, Curr. Sci. 77, 411 (1999); V. Popkov, L. Santen, A. Schadschneider, and G. M. Schütz, J. Phys. A 34, L45 (2001).

[7] F. Spitzer, Adv. Math. 5, 246 (1970).

[8] A. De Masi and P. A. Ferrari, J. Stat. Phys. 38, 603 (1985); R. Kutner and H. van Beijeren, ibid. 39, 317 (1985); D. Dhar, Phase Transit. 9, 51 (1987); S. N. Majumdar and M. Barma, Phys. Rev. B 44, 5306 (1991); L.-H. Gwa and H. Spohn, Phys. Rev. A 46, 844 (1992); B. Derrida, M. R. Evans, and V. Pasquier, J. Phys. A 26, 4911 (1993); D. Kim, Phys. Rev. E 52, 3512 (1995); O. Golinelli and K. Mallick, J. Phys. A 37, 3321 (2004); O. Golinelli and K. Mallick, ibid. 38, 1419 (2005).

[9] B. Derrida, E. Domany, and D. Mukamel, J. Stat. Phys. 69, 667 (1992).

[10] B. Derrida, M. R. Evans, V. Hakim, and V. Pasquier, J. Phys. A 26, 1493 (1993).
[11] G. Schütz and E. Domany, J. Stat. Phys. 72, 277 (1993); G. Schütz, Phys. Rev. E 47, 4265 (1993).

[12] J. Krug, Phys. Rev. Lett. 67, 1882 (1991).

[13] See Refs. [9-11]; See Ref. [14(a)]; M. Dudzinski and G. M. Schütz, ibid. 33, 8351 (2000); Z. Nagy, C. Appert, and L. Santen, J. Stat. Phys. 109, 623 (2002); S. Takesue, T. Mitsudo, and H. Hayakawa, Phys. Rev. E 68, 015103(R) (2003); P. Pierobon, A. Parmeggiani, F. von Oppen, and E. Frey, ibid. 72, 036123 (2005); S. Gupta, S. N. Majumdar, C. Godrèche, and M. Barma, ibid. 76, 021112 (2007); J. de Gier and F. H. L. Essler, J. Stat. Mech. (2006) P12011.

[14] (a) A. B. Kolomeisky, G. M. Schütz, E. B. Kolomeisky, and J. P. Straley, J. Phys. A 31, 6911 (1998); (b) V. Belitzky and G. M. Schütz, Electron. J. Probab. 7, paper No. 11 (2002).

[15] L. Santen and C. Appert, J. Stat. Phys. 106, 187 (2002).

[16] D. A. Adams, R. K. P. Zia, and B. Schmittmann, Phys. Rev. Lett. 99, 020601 (2007).

[17] M. Ha and M. den Nijs, Phys. Rev. E 66, 036118 (2002).

[18] D. A. Adams, B. Schmittmann, and R. K. P. Zia, J. Stat. Mech. (2008) P06009.

[19] T. Chou, Biophys. J. 85, 755 (2003).

[20] L. J. Cook and R. K. P. Zia, J. Stat. Mech. (2009) P02012.

[21] Alternatively, $\Delta \widetilde{\rho}(i \mid m)$ can be regarded as a coarse-grained average of $\Delta \rho(i)$, given by $\sum_{j=0}^{m} \Delta \rho(i+j-m / 2) /(m+1)$, or simply $[\rho(i+1+m / 2)-\rho(i-m / 2)] /(m+1)$.

[22] L. J. Cook, Ph.D. thesis, Virginia Tech, (unpublished).

[23] A. N. Kolmogoroff, Math. Ann. 112, 155 (1936); for a recent discussion, see, e.g., Ref. [25].

[24] L. J. Cook and R. K. P. Zia (unpublished).

[25] R. K. P. Zia and B. Schmittmann, J. Phys. A 39, L407 (2006); J. Stat. Mech. (2007) P07012.

[26] J. J. Dong, Ph.D. thesis, Virginia Tech, 2008 (http:// scholar.lib.vt.edu/theses/available/etd-04092008-113617/ restricted/thesis_V200803_rev4.pdf); see also J. J. Dong et al. in Ref. [4]. 